\title{
Generalized Bayesian Model Selection for Speckle on Remote Sensing Images
}

\author{
Oktay Karakuş $^{\circledR}$, Ercan E. Kuruoğlu, Senior Member, IEEE, and Mustafa A. Altınkaya, Member, IEEE
}

\begin{abstract}
Synthetic aperture radar (SAR) and ultrasound (US) are two important active imaging techniques for remote sensing, both of which are subject to speckle noise caused by coherent summation of back-scattered waves and subsequent nonlinear envelope transformations. Estimating the characteristics of this multiplicative noise is crucial to develop denoising methods and to improve statistical inference from remote sensing images. In this paper, reversible jump Markov chain Monte Carlo (RJMCMC) algorithm has been used with a wider interpretation and a recently proposed RJMCMC-based Bayesian approach, trans-space RJMCMC, has been utilized. The proposed method provides an automatic model class selection mechanism for remote sensing images of SAR and US where the model class space consists of popular envelope distribution families. The proposed method estimates the correct distribution family, as well as the shape and the scale parameters, avoiding performing an exhaustive search. For the experimental analysis, different SAR images of urban, forest and agricultural scenes, and two different US images of a human heart have been used. Simulation results show the efficiency of the proposed method in finding statistical models for speckle.
\end{abstract}

Index Terms-Reversible jump MCMC, speckle noise modeling, SAR imagery, ultrasound imagery, envelope distributions, generalized (heavy-tailed) Rayleigh distribution.

\section{INTRODUCTION}

$\mathbf{R}$ EMOTE sensing imaging is a commonly used imaging method in real life applications where the object of interest is observed without interaction or a direct contact. Remote sensing systems can be divided into two groups, which are passive and active. Passive systems, e.g. airborne photography, utilize the natural source of energy, such as sun light, to gather information from the environment. In contrast, active remote sensing systems have their own energy source and gather information by sending waves and receiving the reflected ones from the surface [1]. The object of interest may

Manuscript received November 18, 2017; revised May 21, 2018 and July 15, 2018; accepted October 13, 2018. Date of publication October 26, 2018; date of current version December 3, 2018. The associate editor coordinating the review of this manuscript and approving it for publication was Dr. Weisheng Dong. (Corresponding author: Oktay Karakuş).

O. Karakuş was with the Department of Electrical-Electronics Engineering, Izmir Institute of Technology, 35430 Izmir, Turkey. He is now with the Visual Information Laboratory, University of Bristol, Bristol BS8 1TH, U.K. (e-mail: o.karakus@bristol.ac.uk).

E. E. Kuruoğlu is with ISTI-CNR, 56124 Pisa, Italy (e-mail: ercan.kuruoglu@isti.cnr.it).

M. A. Altınkaya is with the Department of Electrical-Electronics Engineering, İzmir Institute of Technology, 35430 İzmir, Turkey (e-mail: mustafaaltinkaya@iyte.edu.tr).

Digital Object Identifier 10.1109/TIP.2018.2878322 be as small-scale as a human heart, or sometimes as large-scale as the earth's surface. Examples of remote sensing imaging include radar, sonar, ultrasound imaging.

Synthetic aperture radar (SAR) is one of the active remote sensing imaging methods and has become popular over the last decades due to its applicability in environmental/agricultural monitoring, map making, mine detection, etc. [2]. Additionally, SAR imagery is not affected by weather conditions and it can penetrate soil, clouds and forest canopy [3]. SAR is capable of producing high quality images even under severe conditions compared to other imaging methods such as optical and infrared while avoiding the limitations of these methods, e.g. night-time or cloud-covers.

Ultrasound (US) imaging is also an active remote sensing method which has been utilized both for medical and industrial applications. In standard ultrasound systems, there are three types of measured data, which are radio frequency (RF) signals, envelope-detected signals/images and B-mode images [4]. The resulting display ultrasound image is obtained after several operations: firstly measuring multiple RF signals and converting all these RF signals into an envelope image. Then, this envelope image is log-compressed and post-processed in order to obtain an ultrasound image.

Both SAR and US images are obtained via wave reflection and thus, have very similar characteristics. A common and important problem degrading statistical inference from both SAR and US imagery is the presence of multiplicative speckle noise. The received back-scattered signals sum up coherently and then undergo nonlinear transformations. This in turn causes a granular look in the resulted images, which is referred to as speckle noise [3], [5].

Speckle noise may lead to loss of crucial details in both SAR and US images and cause problems in processing of these images such as in feature detection, segmentation or classification. The first step in dealing with speckle noise, is to determine its statistical characteristics precisely. In both SAR and US imaging, general practice in the early applications in the literature, has been to utilize Rayleigh distribution as the amplitude distribution model, due to its analytical simplicity and central limit theorem, assuming both the real and imaginary parts of the back-scattered wave follow a Gaussian distribution [6], [7].

Despite its popularity in practice, Rayleigh distribution has not been successful in modeling some types of SAR and US images and using other distributions, which exhibit more impulsive behavior, has been more successful in coping 
with the speckle noise effects. Particularly, high resolution urban SAR images [3], [8] and tissue sections of the US images [9]-[11] exhibit impulsive characteristics. Various distributions have been studied in order to represent the impulsive and heavy-tailed behavior of SAR images. Examples can be listed as $\mathcal{K}$-distribution for composite terrains [12], land and sea clutters [13], log-normal distribution for high-resolution sea clutter [14], Weibull for weather and sea clutters [15] and for target detection in background radar clutter [16]. Moreover, in US imaging, examples can be listed as: $\mathcal{K}$-distribution [17] for tissue modeling, Nakagami [7] for classification of breast masses and Gamma based distributions [5] in industrial ceramic US images.

There are other studies which aim to provide a global distribution family for all kind of SAR images or generate a mixture of distributions to obtain the best matching distribution for a given image. Sportouche et al. [18] proposed a mimicking approach which is based on two popular and flexible distributions, namely Fisher and generalized gamma distributions. The main purpose is to use one of these distributions to mimic statistical characteristics of the high-resolution SAR images. It was shown that these two distributions are good selections for a mimicking purpose and high potentials were proposed. Krylov et al. [19] proposed an enhanced dictionary based statistical modeling study of amplitude distributions of very high-resolution SAR images. In that study, eight popular distributions (Nakagami, Weibull, generalized Gamma, etc.) were utilized in the dictionary and an amplitude SAR image was modeled with a mixture pdf of $\mathrm{K}$ components of these distributions.

Reversible jump Markov chain Monte Carlo (RJM$\mathrm{CMC}$ ) algorithm is an extended version of the classical Metropolis-Hastings algorithm and was first introduced by Peter Green [20] as a model selection method. Unlike the Metropolis-Hastings algorithm, the model space dimension has been also defined as a variable, and thus a general method which provides transitions between different dimensional spaces has been proposed. Although Green's formulation is very general, to the best of our knowledge the general usage of the algorithm has been limited to model dimension selection studies of the same classes of models, particularly in model estimation, in analyzing mixture processes. However, the original formulation of RJMCMC exhibits a great potential in exploring different classes of models rather than just being trans-dimensional.

In our recent study [21], we have proposed a generalized framework, trans-space RJMCMC, in order to explore different classes of models where moment-based RJMCMC transitions enable the transfer of the information learned in the most recent model class to another and that interpretation donated RJMCMC with the capability of choosing the most suitable model among different (one-dimensional) impulsive distribution families. The methodology proposed in [21] is highly flexible and can be adjusted to various applications by utilizing different types of moments, such as fractional lower orders, negative orders. In this study, we propose utilizing this methodology (trans-space RJMCMC [21]) in generalized model class selection for remote sensing images of SAR and US. This study is dealing with modeling of the amplitudes of the bivariate distributions, i.e. this modeling is related to $2 \mathrm{D}$ phenomena.

We utilize a model class space which includes various frequently used envelope distribution families in order to represent the statistical characteristics of the images containing speckle noise. For the experimental analysis, different SAR images of urban, forest and agricultural scenes and US images of a human heart have been used. We would like to underline that unlike other studies which restrict their attention to a single family of distributions at a time, our methodology is able to choose from various distribution classes, the library of which can be extended or reduced depending on the needs of the user. Thus, users can develop applications by using the method in this paper, avoiding performing exhaustive searches to estimate the most suitable statistical distribution.

On the other hand, please note that the purpose of this paper is not to propose a universal distribution family for remote sensing images as in [18], or not to demonstrate a mixture modeling of the relevant distributions for SAR images as in [19]. We would like to propose, with this paper, an RJMCMC based Bayesian automatic statistical modeling scheme for remote sensing images of SAR and US. Contrary to the important studies [18], [19], generating a mixture process or proposing a universal distribution for remote sensing images are not in the scope of this paper.

The rest of the paper is organized as follows: trans-space RJMCMC is discussed in Section II. The utilized envelope distribution families and the trans-space RJMCMC methodology are given in Section III. Experimental studies and results are provided in Section IV and Section V concludes the paper with a brief summary.

\section{TRANS-SPACE RJMCMC}

The general practice is to use RJMCMC in model estimation problems such as autoregressive (AR) [22] and autoregressive integrated moving average (ARIMA) [23]. Another popular application of RJMCMC is to analyze the mixtures of distributions such as Gaussian [24] and symmetric $\alpha$-stable ( $\alpha \alpha S)$ mixtures [25].

In the classical "trans-dimensional" RJMCMC approach, we first assume that a transition from a Markov chain $x$ to $x^{\prime}$ with a RJMCMC move, $m$, with probability of $p_{m}$ is proposed. This transition will be accomplished with a relation (or namely a reversible function), $h$, between the parameter vectors of states $x$ and $x^{\prime}$, which are $\boldsymbol{\theta}$ and $\boldsymbol{\theta}^{\prime}$. Since the dimension matching between the parameter spaces is satisfied, this transition is accepted with a probability of:

$$
A\left(x \rightarrow x^{\prime}\right)=\min \left\{1, \frac{\pi\left(x^{\prime}\right) p_{m_{\mathrm{R}}} \chi\left(\mathbf{u}^{\prime}\right)}{\pi(x) p_{m} \chi(\mathbf{u})}\left|\frac{\partial h(\boldsymbol{\theta}, \mathbf{u})}{\partial(\boldsymbol{\theta}, \mathbf{u})}\right|\right\},
$$

where $\pi(\cdot)$ refers to the target distribution of interest, $\chi(\cdot)$ are the distributions for the auxiliary variable vectors $\mathbf{u}$ and $\mathbf{u}^{\prime}$, respectively which are required to provide dimension matching for the moves $m$ and its reversible move $m_{\mathrm{R}}$. The term $\left|\frac{\partial h(\boldsymbol{\theta}, \mathbf{u})}{\partial(\boldsymbol{\theta}, \mathbf{u})}\right|$ is the magnitude of the Jacobian. 
Despite having various application areas, all the studies mentioned above have utilized RJMCMC in a limited perspective, particularly, within the same classes of models and trans-dimensional cases. However, the original formulation of Green lends itself to a much wider interpretation than just exploring spaces of different dimensions. In our previous studies (e.g. [26]), we have utilized RJMCMC in exploring linear and nonlinear model spaces of nonlinear time series models, polynomial AR (PAR), polynomial MA (PMA) and polynomial ARMA (PARMA). In addition, a Volterra system identification study has been performed in [27].

The recently proposed "trans-space" RJMCMC in [21] lets us to explore different generic model classes instead of focusing on the parameter dimension. Defining transitions over a "common feature" such as moment of different model spaces in the trans-space approach is a mandatory choice for the algorithm not to start the search from the scratch in the jumped model space. The approach in [21] utilizes fractional lower ordered moments (FLOMs) as common feature and perform transitions between spaces of different probability distribution families, namely impulsive distributions. In this paper, a trans-space RJMCMC approach with first order negative moment-based transitions which explores spaces of envelope (or amplitude) distribution families, has been used. The original acceptance ratio expression in (1) is still valid and is adapted according to the RJMCMC moves proposed in this study.

\section{TRAns-SpaCe RJMCMC For SPECKLE NOISE MODELING}

\section{A. Envelope Distribution Families}

Envelope distribution [28] families are continuous distribution families which are supported on the interval, $(0, \infty)$. Due to the nature of active remote sensing systems, the amplitude distributions occur via coherent addition of the reflected waves, and hence, the speckle noise of SAR and ultrasound images has been modeled by using distributions of this type. In this paper, five different envelope distributions, which are widely used in modeling speckle noise of SAR and US images from various environments, have been investigated. These are Nakagami, $\mathcal{K}$, Weibull, Gamma and generalized Rayleigh distributions.

1) Nakagami Distribution Family: For remote sensing applications, Nakagami distribution has been utilized in classification/characterization [29] and speckle suppression of US images [30] and speckle modeling of agricultural and mountain scene SAR images [31]. The univariate Nakagami distribution pdf can be defined as [32]:

$$
f(x)=\frac{2 \alpha^{\alpha}}{\Gamma(\alpha) \gamma^{\alpha}} x^{2 \alpha-1} \exp \left(-\frac{\alpha}{\gamma} x^{2}\right)
$$

where $\Gamma(\cdot)$ is the gamma function, $\alpha \geq 0.5$ refers to the shape parameter and $\gamma>0$ represents the scale parameter of the Nakagami distribution family. One-sided Gaussian distribution and Rayleigh distribution are the special members of Nakagami distribution family for shape parameters of 0.5 and 1 , respectively. The $p$ th order moment of the Nakagami distribution is [33]:

$$
E\left(\mathbf{x}^{p}\right)=\frac{\Gamma\left(\alpha+\frac{p}{2}\right)}{\Gamma(\alpha)}\left(\frac{\gamma}{\alpha}\right)^{p / 2} .
$$

2) $\mathcal{K}$-Distribution Family: For remote sensing applications, $\mathcal{K}$-distribution has been utilized in modeling composite terrain SAR images [12], land and sea radar clutters [13], and US imagery [9].

$\mathcal{K}$-distribution is a distribution family which arises by compounding two gamma distributions. Assume a random variable $x$ is Gamma distributed with mean $m$ and shape $L$, the mean $m$ of which is also a Gamma distributed random variable. Thus, $x$ is $\mathcal{K}$-distributed. Additionally, $\mathcal{K}$-distribution is also a product distribution. Particularly, it is the distribution of a random variable which is the product of two independent Gamma random variables. The pdf of a univariate $\mathcal{K}$-distribution can be defined as [34]:

$$
f(x)=\frac{2}{\gamma \Gamma(\alpha+1)}\left(\frac{x}{2 \gamma}\right)^{\alpha+1} K_{\alpha}\left(\frac{x}{\gamma}\right)
$$

where $K_{\alpha}$ refers to the modified Bessel function of order $\alpha$ and $\alpha$ and $\gamma>0$ represent the shape and the scale parameters, respectively. The $p$ th order moment of the $\mathcal{K}$-distribution is [34]:

$$
E\left(\mathbf{x}^{p}\right)=\frac{2^{p} \Gamma\left(\frac{p}{2}+1\right) \Gamma\left(\alpha+1+\frac{p}{2}\right)}{\Gamma(\alpha+1)} \gamma^{p} .
$$

3) Weibull Distribution Family: For remote sensing applications, Weibull distribution has been used to model land (forest, rocky mountains), sea and sea ice radar clutters [15] and cardiac US images [35]. A univariate Weibull distribution pdf can be defined as [36]:

$$
f(x)=\frac{\alpha}{\gamma}\left(\frac{x}{\gamma}\right)^{\alpha-1} \exp \left(-\left(\frac{x}{\gamma}\right)^{\alpha}\right)
$$

where $\alpha$ is the shape and $\gamma>0$ is the scale parameter. Weibull distribution family has special members for $\alpha=1$ and $\alpha=2$, which are the well-known exponential and Rayleigh distributions, respectively. The $p$ th order moment of the Weibull distribution is [36]:

$$
E\left(\mathbf{x}^{p}\right)=\Gamma\left(1+\frac{p}{\alpha}\right) \gamma^{p} .
$$

4) Gamma Distribution Family: In remote sensing applications, Gamma distribution has been used to model sea [37] and land [8] SAR images, and ultrasound speckle noise [5]. The univariate gamma distribution pdf can be expressed as [36]:

$$
f(x)=\frac{x^{\alpha-1}}{\gamma^{\alpha} \Gamma(\alpha)} \exp \left(-\frac{x}{\gamma}\right)
$$

where $\alpha$ refers to the shape parameter and $\gamma>0$ is the scale parameter. The well-known exponential and chi-squared distributions are special members of the gamma distribution family. The $p$ th order moment of the Gamma distribution is [36]:

$$
E\left(\mathbf{x}^{p}\right)=\frac{\Gamma(\alpha+p)}{\Gamma(\alpha)} \gamma^{p} .
$$




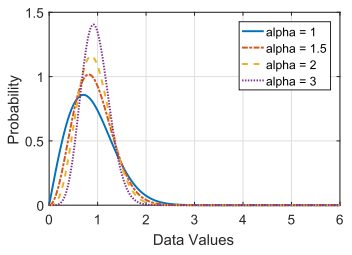

(a)

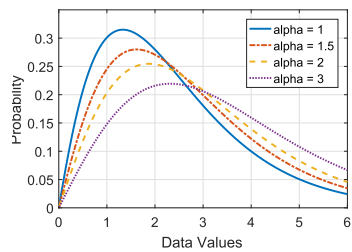

(b)

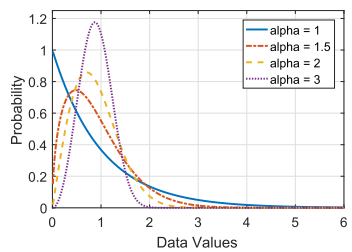

(c)

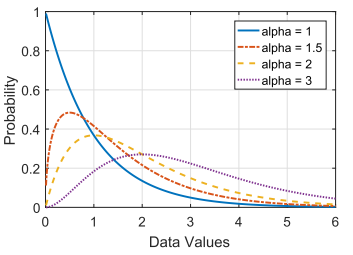

(d)

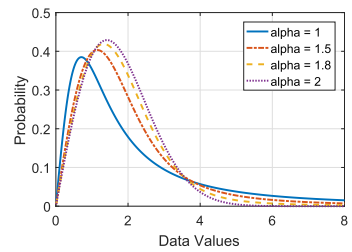

(e)

Fig. 1. Pdf of envelope distribution families for different values of the shape parameter $\alpha$. For all the families the scale parameter, $\gamma=1$. (a) Nakagami. (b) $\mathcal{K}$. (c) Weibull. (d) Gamma. (e) Generalized Rayleigh.

5) Generalized Rayleigh Distribution: Generalized (heavy tailed) Rayleigh distribution [3] has been proposed in order to model urban scenes SAR images with a better performance than the classical envelope distributions Rayleigh, Weibull and $\mathcal{K}$. The classical Rayleigh distribution represents the distribution of a random variable which is the magnitude of a 2 dimensional vector, components of which are zero mean, equal variance Gaussian random variables. Generalized Rayleigh distribution represents the distribution of a random variable which is again a 2-dimensional vector. However this time, the components of the vector are zero location equal dispersion $\mathrm{S} \alpha \mathrm{S}$ random variables. Generalized Rayleigh distribution has a pdf expression in integral form as [3]:

$$
f(x)=x \int_{0}^{\infty} s \exp \left(-\gamma s^{\alpha}\right) J_{0}(s x) d s
$$

where $\gamma>0$ is the scale parameter, $0<\alpha \leq 2$ is the shape parameter and $J_{0}(\cdot)$ refers to the zeroth order Bessel function of the first kind. Rayleigh distribution is the special member of generalized Rayleigh distribution with $\alpha=2$. The $p$ th order moment of the generalized Rayleigh distribution, for $-1.5<$ $p<-0.5$, is as follows [3]:

$$
E\left(\mathbf{x}^{p}\right)=\frac{2^{p+1} \Gamma\left(\frac{p}{2}+1\right) \Gamma\left(\frac{-p}{\alpha}\right)}{\alpha \Gamma\left(\frac{-p}{2}\right)} \gamma^{p / \alpha} .
$$

In Figure 1, densities for Nakagami, $\mathcal{K}$, Weibull, Gamma and generalized Rayleigh distributions are shown, respectively. For Nakagami densities increasing the shape parameter $\alpha$ makes the densities peaky and heavy tailed relative to the ones with smaller $\alpha$. Weibull distribution also follows very similar characteristics to Nakagami distribution. For $\mathcal{K}$ and Gamma distributions, decreasing the shape parameter makes them more peaky and heavy tailed than the ones with larger $\alpha$. For generalized Rayleigh distributions, tail probabilities decrease as order of $\alpha$ values increases, whereas peak values are very similar for all the $\alpha$ values. Generalized Rayleigh, $\mathcal{K}$ and Gamma distributions are distinguished from Nakagami and Weibull due to heavier tails. Generally, Nakagami and Weibull distributions are less heavy tailed envelope distributions and densities diminish towards zero faster than the other distributions.

\section{B. Implementation of Trans-Space RJMCMC}

The parameter space for RJMCMC contains the shape and the scale parameters of the distributions and also the family identifier parameter which represent the families. Particularly, the parameter space is formed as: $\boldsymbol{\theta}=\{k, \alpha, \gamma\}$ where the family identifier $k$ refers to Nakagami, $\mathcal{K}$, Weibull, generalized Rayleigh and Gamma for values between 1 and 5, respectively.

The joint posterior density, or namely RJMCMC target distribution of interest, $f(\boldsymbol{\theta} \mid \mathbf{x})$, can be written from Bayes Theorem as:

$$
f(\boldsymbol{\theta} \mid \mathbf{x}) \propto f(\mathbf{x} \mid k, \alpha, \gamma) f(\alpha \mid k) f(k) f(\gamma)
$$

where $f(\mathbf{x} \mid k, \alpha, \gamma)$ is the likelihood and corresponds to Nakagami, $\mathcal{K}$, Weibull, generalized Rayleigh and Gamma pdfs for values of $k, 1,2,3,4$ and 5 , respectively.

Priors are selected as the following:

$$
\begin{aligned}
f(\gamma) & =1 / \gamma \\
f(k) & =1 / 5, \quad \forall k, \\
f(\alpha \mid k) & =\mathcal{U}\left(B_{\text {low }}^{(k)}, B_{\text {up }}^{(k)}\right) \quad \forall k,
\end{aligned}
$$

where $\mathcal{U}(\cdot)$ represent a uniform distribution, $B_{\text {low }}^{(k)}$ is the lower bound and equals to $0.5,0.5,1,0.5,1$ for values of $k$ from 1 to 5 , respectively. Additionally, $B_{\mathrm{up}}^{(k)}$ refers to the upper bound and equal to $2,10,5,2,6$. Please note that this selection for the bounds is not unique and both of the bounds are selected intuitively in order to cover lots of distributions in the families. Increasing upper bounds does not have any meaningful effect on the results for this study. Interested users can use different upper bounds for different data sets. Since it is not possible to define a common conjugate prior for the scale parameter of all candidate distributions, we choose a noninformative (Jeffrey's) prior distribution for $\gamma$ in this study. In addition, all possible distribution families are selected as equiprobable each of which has a probability of $1 / 5$.

1) Model Moves: In this study, two main RJMCMC moves are defined to perform transitions between different probability distribution families, which are life and switch moves. The life move performs the classical $\mathrm{MH}$ algorithm and is used to update the scale parameter, $\gamma$, while the algorithm remains in the same family and at the same shape parameter $\left(k^{\prime}=k\right.$ and $\alpha^{\prime}=\alpha$ ). Two different switch moves are defined and utilized for RJMCMC transitions, which are within the same family and between different families. These two different switch moves [21] are named as intra-class-switch and inter-classswitch. Intra-class-switch move is performed within the same family $\left(k^{\prime}=k\right)$ whereas the inter-class-switch move performs switching to a different family $\left(k^{\prime} \neq k\right)$.

For both of the switch moves, model transitions must be handled carefully. In statistics, moments are very important in having an idea about a probability distribution such as its mean and variance. Moment-based parameter estimation methods (namely Method-of-Moments (MoM)) have been widely used 
for the candidate distribution classes in this study, which can be listed as for Weibull [38], Nakagami [39], Gamma [40], $\mathcal{K}$-distribution [34] and generalized Rayleigh [3]. Since the $p$ th order moments are well defined with some constraints for all the candidate distribution families, using it as the "common feature" between distributions is a very convenient option for our purpose. This feature can be thought of as being a super parameter defined for every class boiling individual different parameters of each class into a common one. Please note that using $p$ th order moments in the proposed method is not a unique choice. Interested readers can utilize other statistics, such as log-moments. On the other hand, please also note that one can create a setup with blind proposals between different classes avoiding using MoM based approach in this paper. However, using a common parameter like moments, implements a highly efficient proposal mechanism for RJMCMC and provides faster convergence of the algorithm reducing the possibility of having local traps during the Markov chains.

The logic behind the moment-based transitions, is to hold the $p$ th order moment fixed for both the most recent and the candidate distributions during a switch move. For example, given data $x$, for an inter-class-switch move from distribution family $k$ with parameters $\alpha$ and $\gamma$ to family $k^{\prime}$, the candidate distribution's shape parameter $\alpha^{\prime}$ is proposed from a proposal distribution and the scale parameter $\gamma^{\prime}$ is calculated, providing the following expression:

$$
E_{k}\left(\mathbf{x}^{p}\right)=E_{k^{\prime}}\left(\mathbf{x}^{p}\right) .
$$

Thus, the information learned in the most recent distribution is transferred to the new distribution. For intra-class-switch move, it is straightforward to show that both sides of the expression in (16) belong to the same family with different shape parameters.

Up to this point we have discussed the motivation for why and how the moment based transitions should be performed. Another important point is the choice of the moment degree " $p$." Examining the moment definitions of the families in Section III-A, we can clearly see that the generalized Rayleigh family only has moments for $p$ values between -1.5 and -0.5 . This constraint forces using negative moments for all the distributions, and common-feature based transitions are performed over negative order moments. In order to eliminate some possible degradations that may arise from using fractional moments, $p=-1$ is the best choice and have been used in this study. Existence of the first negative moment has been tested according to the study in [41] and this selection $(p=-1)$ is valid for generalized Rayleigh and $\mathcal{K}$ without any constraint on selection of the shape and the scale parameters. Weibull and Gamma have a constraint of $\alpha>1$ and Nakagami has first order negative moments for $\alpha>0.5$. All these constraints are satisfied with a bounded selection for shape parameters in (15).

Figure 2 depicts the flow diagram of the proposed usage of RJMCMC in speckle noise modeling of remote sensing images. Details of the RJMCMC moves are given in the sequel.

2) Proposal Distributions for Moves: As stated above, life move updates only the scale parameter, $\gamma$ where $k$ and $\alpha$

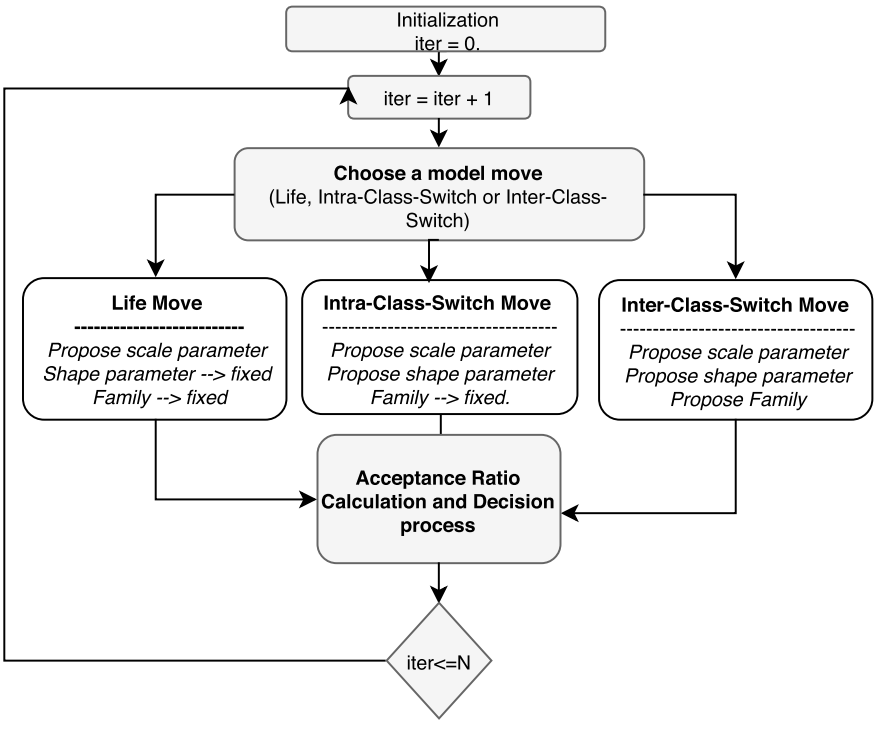

Fig. 2. Flow Diagram for the Proposed method.

remain fixed. For this purpose, we decided to use a proposal distribution for life move as follows:

$$
q\left(\gamma^{\prime} \mid \gamma\right)=\mathcal{T} \mathcal{N}\left(\gamma, \xi_{\text {scale }}\right) \text { for interval }(0, \infty)
$$

where $\mathcal{T} \mathcal{N}\left(\gamma, \xi_{\text {scale }}\right)$ refers to a Gaussian distribution where its mean $\gamma$ is the most recent value of the scale parameter, and its variance is $\xi_{\text {scale }}$. This Gaussian distribution is truncated to lie within the interval of $(0, \infty)$ afterwards by rejecting samples outside this interval. This truncation procedure aims to satisfy the condition $\gamma>0$.

For both of the switch moves, the sampling of $\alpha$ is performed firstly. The range of $\alpha$ is discretized by increments of 0.05 in order to avoid numerical errors during the simulations. A discretized Laplace $(\mathcal{D} \mathcal{L}(\cdot))$ and a uniform distribution are selected as proposal distributions for intra and inter-class switch moves, respectively. The reason for a $\mathcal{D} \mathcal{L}(\cdot)$ selection for intra-class-switch is to make the samples come from a distribution that is symmetrical around the most recent value and heavier tailed than a Gaussian. On the other hand, during a transition between different families, we have limited prior information for the shape parameter, $\alpha$. Hence, all $\alpha$ values belonging to the new family are assumed as equiprobable and a uniform proposal distribution is selected for inter-class-switch move.

Scale parameter $\gamma$ transitions is performed via the moment based method which is mentioned above via functions $g(\cdot)$ and $w(\cdot)$ for intra and inter-class-switch moves, respectively. These functions are obtained provided that the equality in (16) holds. An example derivation for a transition from Weibull distribution $(k=3, \gamma, \alpha)$ to Gamma $\left(k=5, \gamma^{\prime}, \alpha^{\prime}\right)$ is given below

$$
\begin{aligned}
E_{3}\left(\mathbf{x}^{-1}\right) & =E_{5}\left(\mathbf{x}^{-1}\right) \\
\Gamma\left(1-\frac{1}{\alpha}\right) \gamma^{-1} & =\frac{\Gamma\left(\alpha^{\prime}-1\right)}{\Gamma\left(\alpha^{\prime}\right)}\left(\gamma^{\prime}\right)^{-1} \\
\gamma^{\prime} & =w\left(\alpha, \alpha^{\prime}, \gamma\right)=\gamma \frac{\Gamma\left(\alpha^{\prime}-1\right)}{\Gamma\left(\alpha^{\prime}\right) \Gamma\left(1-\frac{1}{\alpha}\right)} .
\end{aligned}
$$


It is straightforward to derive all other expressions for $g(\cdot)$ and $w(\cdot)$ functions.

Consequently, proposals for intra-class-switch move and for inter-class-switch move are:

$$
\begin{aligned}
& \alpha^{\prime} \sim \mathcal{D} \mathcal{L}(\alpha, 0.5), \\
& \gamma^{\prime}=g\left(\alpha, \alpha^{\prime}, \gamma\right),
\end{aligned}
$$

and

$$
\begin{aligned}
& \alpha^{\prime} \sim \mathcal{U}\left(B_{\text {low }}^{\left(k^{\prime}\right)}, B_{\mathrm{up}}^{\left(k^{\prime}\right)}\right), \\
& \gamma^{\prime}=w\left(\alpha, \alpha^{\prime}, \gamma\right),
\end{aligned}
$$

respectively.

3) Acceptance Ratio for the Moves: The acceptance ratio expressions obtained for life, intra-class-switch and inter-classswitch moves are as follows:

$$
\begin{aligned}
A_{\text {life }} & =\min \left\{1, \frac{f\left(\mathbf{x} \mid k^{\prime}, \alpha^{\prime}, \gamma^{\prime}\right)}{f(\mathbf{x} \mid k, \alpha, \gamma)} \frac{f\left(\gamma^{\prime}\right)}{f(\gamma)} \frac{q\left(\gamma \mid \gamma^{\prime}\right)}{q\left(\gamma^{\prime} \mid \gamma\right)}\right\} \\
A_{\text {intra-cl-sw }} & =\min \left\{1, \frac{f\left(\mathbf{x} \mid k^{\prime}, \alpha^{\prime}, \gamma^{\prime}\right)}{f(\mathbf{x} \mid k, \alpha, \gamma)} \frac{f\left(\gamma^{\prime}\right)}{f(\gamma)}|J|\right\}, \\
A_{\text {inter-cl-sw }} & =\min \left\{1, \frac{f\left(\mathbf{x} \mid k^{\prime}, \alpha^{\prime}, \gamma^{\prime}\right)}{f(\mathbf{x} \mid k, \alpha, \gamma)} \frac{f\left(\gamma^{\prime}\right)}{f(\gamma)} \frac{f(\alpha \mid k)}{f\left(\alpha^{\prime} \mid k^{\prime}\right)}|J|\right\}
\end{aligned}
$$

where $|J|$ is the magnitude of the Jacobian.

\section{EXPERIMENTAL STUdy}

Experimental studies in this paper consist of two different simulations which are synthetically generated and real data simulations. Results are demonstrated in figures and tables for both simulation scenarios. RJMCMC has been run for 3000 and 10000 iterations for synthetical and real data simulations, respectively. During the parameter estimation stage, only the last half of the iterations (1500 and 5000) have been used and the first half of the iterations are discarded as burn-in period. The initial distribution of the algorithm is a Rayleigh distribution $\left(k^{(0)}=3\right.$ and $\left.\alpha^{(0)}=2\right)$. All three RJMCMC moves are assumed to be equiprobable providing $P_{\text {life }}+P_{\text {intra-cl-sw }}+P_{\text {inter-cl-sw }}=1$. Performance of the fitted distributions to the given data sets has been measured by using two popular statistical significance tests, namely KullbackLeibler (KL) divergence and Kolmogorov-Smirnov (KS) statistics. KL divergence has been used to test the performance by considering the estimated and empirical pdfs, whereas KS statistics and its corresponding $p$-values have been calculated by using the estimated and the empirical CDFs.

\section{A. Synthetically Generated Data Simulations}

In synthetically generated data simulations, five different distributions from each candidate family has been selected. For each selected distribution, 20 different data sets with 1000 samples have been generated and the proposed method have been run to estimate distributions given the data. Selected distributions and their estimation results are given in Table I. Please note that the estimated parameters $(\hat{\alpha}$ and $\hat{\gamma})$ and statistical measures are averages over 20 repetitions.

Examining the results in Table I, all families have been estimated without error via the proposed method.
TABLE I

Modelling Results and Statistical Significance of the ESTIMATES FOR SYNTHETICALLY GENERATED DATA SETS

\begin{tabular}{llccccc}
\hline Family $(\alpha, \gamma)$ & Est. & $\begin{array}{l}\text { Est. } \\
\text { Shape }(\hat{\alpha})\end{array}$ & $\begin{array}{l}\text { Est. } \\
\text { Scale }(\hat{\gamma})\end{array}$ & KL Div. & $\begin{array}{l}\text { KS } \\
\text { Score }\end{array}$ & $\begin{array}{l}\text { KS } \\
p \text {-value }\end{array}$ \\
\hline Nakagami $(1.5,10)$ & Nakagami & 1.5093 & 9.9836 & 0.0314 & 0.0248 & 1.0000 \\
$\mathcal{K}(1.2,5)$ & $\mathcal{K}$ & 1.3948 & 4.8747 & 0.0291 & 0.0231 & 1.0000 \\
Weibull $(4,0.2)$ & Weibull & 3.9407 & 0.1983 & 0.0324 & 0.0235 & 1.0000 \\
GenRayl $(1.7,1)$ & GenRayl & 1.6996 & 1.0512 & 0.0160 & 0.0532 & 1.0000 \\
Gamma $(3.8,0.2)$ & Gamma & 3.6504 & 0.1925 & 0.0292 & 0.0235 & 1.0000 \\
\hline
\end{tabular}

Estimated distribution parameters are also very close to the correct values. These successful estimation results are statistically supported with the KL and KS measures. Examining the measures presented in the table, it can be easily seen that all the distribution estimates are statistically significant for given data sets with $p$-values of 1.0000 .

Parallel MCMCs vs. Trans-Space RJMCMC: In this section, the proposed method has been compared to the exhaustive search of using separate MCMCs for each family in order to evaluate its computational gain in terms of simulation time. The reference method utilizes the proposed method without inter-class-switch moves. For each given data, the reference method performs estimation for each candidate family. Then, the statistical significance measures are calculated for each estimation and the one with minimum measures are selected as the best fitting distribution. All the time cost simulations have been run on a computer with Intel dual core i5-2410M $2.30 \mathrm{GHz}$ processor and 4GB of RAM.

For the simulations, distributions utilized in the previous part (Table I first column) are utilized again. Please note that, for time cost simulations, only one data set has been generated from each family. Simulations run 3000 iterations. The first half of the simulations are discarded as burn-in period in parameter estimation step. For the reference method, move probabilities are selected as equiprobable $\left(P_{\text {life }}=P_{\text {intra-cl-sw }}=0.5\right.$, where $P_{\text {inter-cl-sw }}=0$ ).

Time cost simulation results are presented in Table II. Other than the case of generalized Rayleigh data, the proposed method offers significant time gain compared to separate MCMCs. Actually, the time cost of the MCMCs for the considered distributions are comparable with the exception of the generalized Rayleigh distribution which is significantly more expensive due to the requirement of performing numerical integration for its pdf (given in (10)). This in turn makes the total cost of the reference method, on the order of the cost for the MCMC with generalized Rayleigh data. However, the proposed method avoids most of those time costly visits since it jumps to the states other than the asymptotic distribution of the Markov chain only occasionally after the burn in period [21]. The exception is the case when the data is generalized Rayleigh as shown in the fourth row of Table II. Essentially, the time cost of the proposed method is comparable to a single MCMC and one can expect increased superiority of the proposed method for a set-up including lots (more than five) of candidate models.

Examining the model estimation results and statistical significance measures in Table II, it can be easily seen that, 
TABLE II

PARALLEL MCMC vs. RJMCMC. COMPARISON FOR SimUlation TIME AND ACCURACY

\begin{tabular}{|c|c|c|c|c|c|c|c|c|c|c|c|c|c|c|c|}
\hline \multirow[b]{2}{*}{$\operatorname{Family}((\alpha, \gamma)$} & \multirow[b]{2}{*}{$\begin{array}{l}\text { Total time } \\
T_{1}(\mathrm{sec})\end{array}$} & \multirow[b]{2}{*}{$\begin{array}{l}\text { Est. } \\
\text { Family }\end{array}$} & \multicolumn{3}{|c|}{ The reference method } & \multicolumn{10}{|c|}{ The proposed method } \\
\hline & & & $\begin{array}{l}\text { Est. } \\
\text { Shape }(\hat{\alpha})\end{array}$ & $\begin{array}{l}\text { Est. } \\
\text { Scale }(\hat{\gamma})\end{array}$ & KL Div. & $\begin{array}{l}\text { KS } \\
\text { Score }\end{array}$ & $\begin{array}{l}\text { KS } \\
\text { p-value }\end{array}$ & $\begin{array}{l}\text { Total time } \\
T_{2} \text { (sec) }\end{array}$ & $\begin{array}{l}\text { Est. } \\
\text { Family }\end{array}$ & $\begin{array}{l}\text { Est. } \\
\text { Shape }(\hat{\alpha})\end{array}$ & $\begin{array}{l}\text { Est. } \\
\text { Scale }(\hat{\gamma})\end{array}$ & KL Div. & $\begin{array}{l}\text { KS } \\
\text { Score }\end{array}$ & $\begin{array}{l}\text { KS } \\
\text { p-value }\end{array}$ & $\begin{array}{l}\text { Simulation time } \\
\text { gain }\left(T_{1} / T_{2}\right)\end{array}$ \\
\hline Nakagami $(1.5,10)$ & 5720.54 & Nak: & 1.5125 & 9.7605 & 0.0240 & 0.0126 & 1.0000 & 346.84 & $\mathrm{Nal}$ & 1.5468 & 9.7756 & 0.0235 & 0.0142 & 1.0000 & 16.49 \\
\hline $\mathcal{K}(1.2,5)$ & 6240.30 & $\mathcal{K}$ & 1.7526 & 4.5215 & 0.0183 & 0.0104 & 1.0000 & 465.36 & $\mathcal{K}$ & 1.8553 & 4.4349 & 0.0181 & 0.0121 & 1.0000 & 13.41 \\
\hline Weibull $(4,0.2)$ & 7170.83 & Weibull & 4.0893 & 0.1983 & 0.0236 & 0.0125 & 1.0000 & 558.63 & Weibull & 4.0214 & 0.1978 & 0.0252 & 0.0188 & 1.0000 & 12.84 \\
\hline GenRayl $(1.7,1)$ & 6563.70 & GenRayl & 1.6995 & 1.0357 & 0.0165 & 0.0275 & 1.0000 & 5244.45 & GenRayl & 1.6996 & 1.0192 & 0.0171 & 0.0313 & 1.0000 & 1.25 \\
\hline $\operatorname{Gamma}(3.8,0.2)$ & 6112.07 & Gamma & 3.9354 & 0.2077 & 0.0261 & 0.0262 & 1.0000 & 462.01 & Gamma & 3.9599 & 0.2090 & 0.0260 & 0.0268 & 1.0000 & 13.23 \\
\hline
\end{tabular}

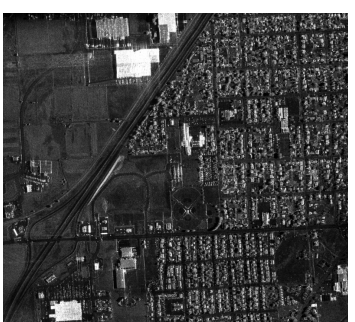

(a)

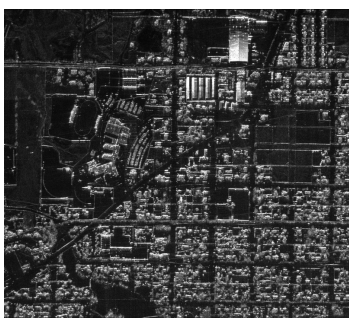

(b)

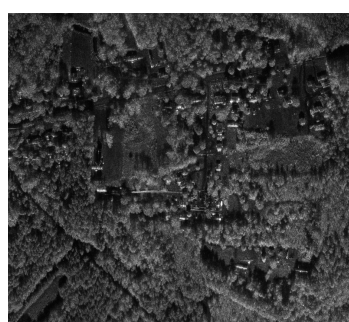

(c)

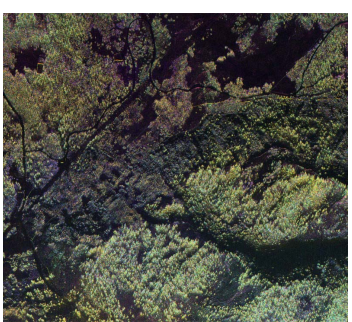

(d)

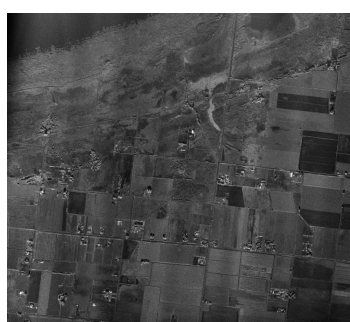

(e)

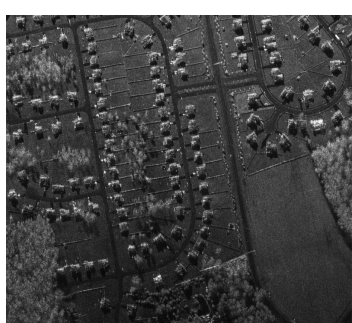

(f)

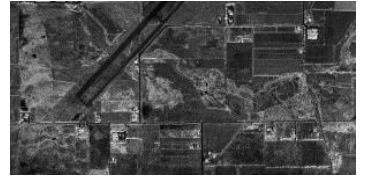

(g)

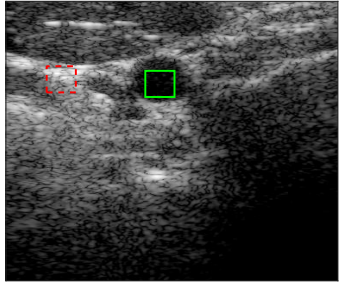

(h)

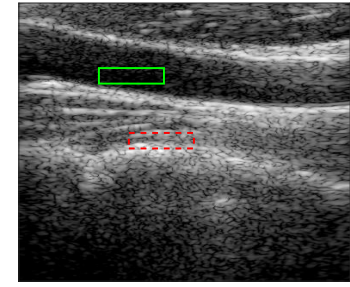

(i)

Fig. 3. SAR and US images. For US images, sections inside solid lines refer to blood sections, whereas sections inside dashed lines refer to tissue sections. (a) SAR-Urban-X. (b) SAR-Urban-L. (c) SAR-Forest-X. (d) SAR-Forest-UHF. (e) SAR-Agricultural-X. (f) SAR-Mixed-X. (g) SAR-Agricultural-L. (h) US-Cross. (i) US-Long.

the reference method and the proposed method perform nearly the same and estimate the same distributions for each of the given data sets. Combining these estimation results with the total simulation times, the proposed method's success can be easily pointed out.

\section{B. Real Data Simulations}

In this study seven different SAR images from urban, forest, agricultural and mixed scenes have been utilized in order to show the statistical differences according to the environment. For each scene, two (one for mixed scene) images have been used which are measured in different frequency bands, X, $\mathrm{L}$ and UHF. All the figures are in intensity format and their sizes are $715 \times 800$ except Agricultural-L the size of which is $150 \times 300$. Particularly, X-band SAR images have been generally used in airborne systems and for terrain mapping. Its frequency range is $8-12 \mathrm{GHz}$ with a wavelength range of 2.5-3.75 cm. L-band SAR images have been utilized in American and Japanese satellites and NASA airborne. Its frequency range is lower than $\mathrm{X}$-band $(1-2 \mathrm{GHz}$ with a wavelength range of $15-30 \mathrm{~cm}$ ). UHF band, also known as P-band, has been used especially for extraterrestrial targets and has the lowest frequency band compared to $\mathrm{X}$ an L bands which is $300 \mathrm{MHz}-1 \mathrm{GHz}$ [42]. All the SAR images in this study are downloaded from [43] and are shown in Figure 3. For the simulation issues, in order to reduce the computational load and removing the effects of the homogenous areas on the images, all the SAR images are down-sampled with a factor of 5. Originally, the image in Figure 3 (d) is colored, however it is transformed into a gray-scale image before simulations by averaging its red, green and blue components.

US images used in this study, are plane-wave imaging challenge in medical ultrasound (PICMUS) data sets [44] containing two measurements from a volunteer, which is recorded on the carotid artery. A 75 plane-wave sequence was recorded with a Verasonics Vantage 256 research scanner and an L11 probe (Verasonics Inc., Redmond, WA). The difference between the two images are their sections of interest, one of them is cross-sectional, the other one is longitudinal (For the details of the data and the PICMUS challenge please see [44]). For both of the images, two rectangular speckle patches are extracted from the envelope image, belonging to tissue and blood pool sections. Each extracted section comprises around 2000 samples. Please note that all the analysis for these sections has been performed on raw data (before down-sampling and log-compression). In Figure 3(h)-(i), the two aforementioned US images of cross and longitudinal sections are shown, respectively. Rectangles with solid and dashed lines refer to the extracted blood and tissue sections, respectively.

For all the SAR and US data sets, simulations have been repeated 20 times and averages over these repetitions have been presented in the tables. In real data simulations, in order 


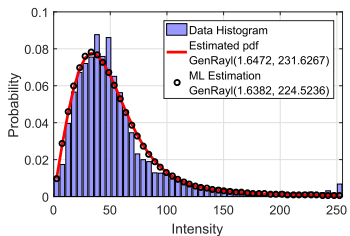

(a)

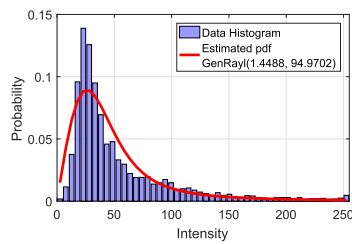

(c)

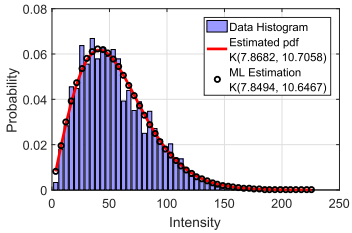

(e)

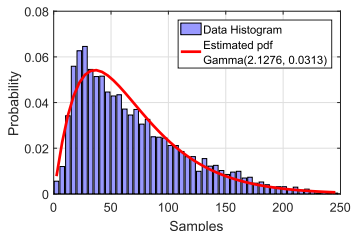

(g)

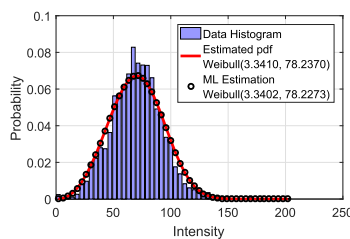

(i)

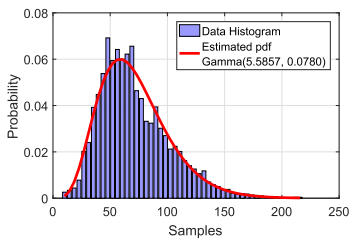

(k)

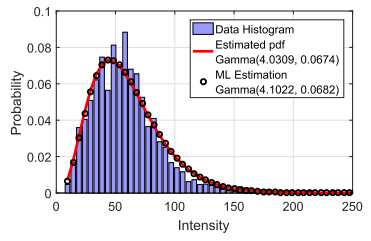

(m)

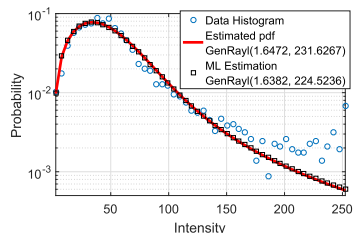

(b)

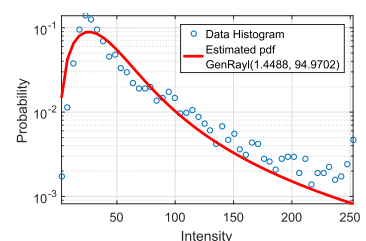

(d)

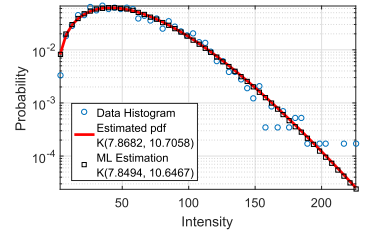

(f)

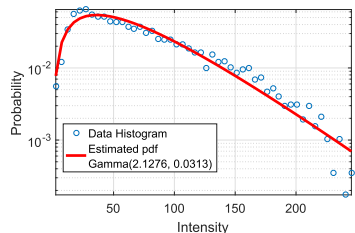

(h)

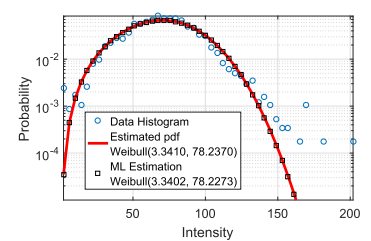

(j)

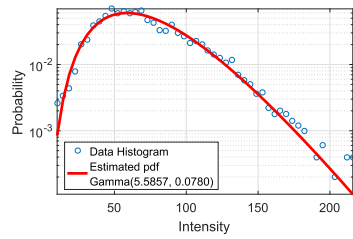

(1)

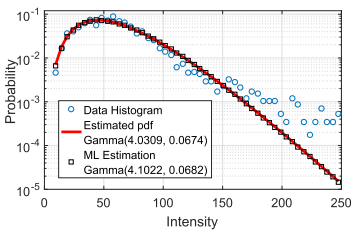

(n)
Fig. 4. SAR image distribution modeling results. Sub-figures on the left column refer to the estimated pdfs in linear scale, whereas the ones on the right column are in logarithmic scale. (a)-(b) Urban-X. (c)-(d) Urban-L. (e)-(f) Forest-X. (g)-(h) Forest-UHF. (i)-(j) Agricultural-X. (k)-(1) Agricultural-L. (m)-(n) Mixed-X.

to show the effectiveness of the proposed method, we have also performed a maximum likelihood (ML) based parameter estimation method for all the families. The ML estimated

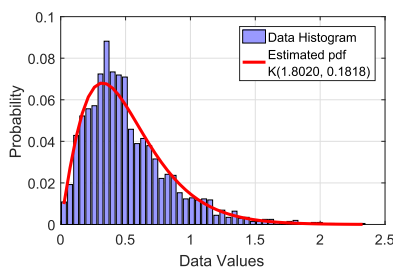

(a)

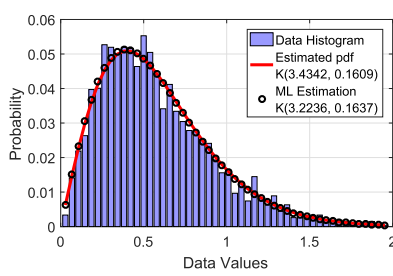

(c)

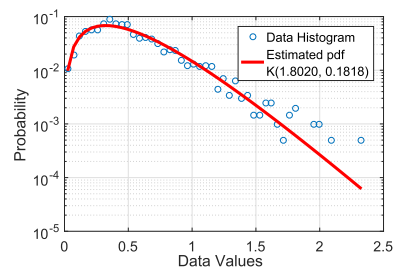

(e)

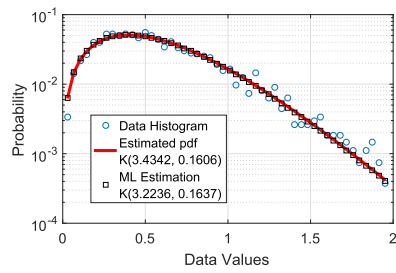

(g)

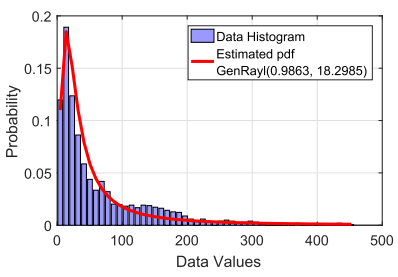

(b)

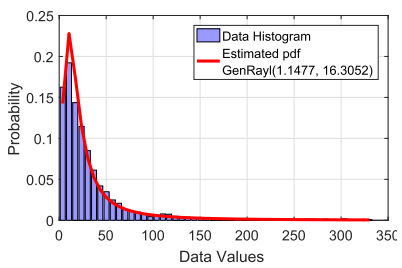

(d)

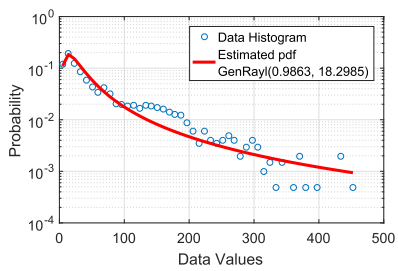

(f)

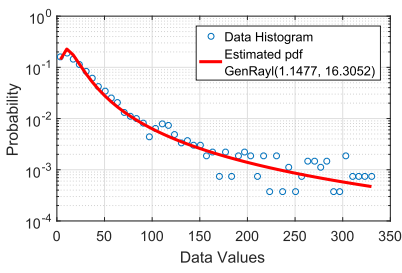

(h)
Fig. 5. US image distribution modeling results. (a)-(d): Estimated pdfs in linear scale, (e)-(h): Estimated pdfs in logarithmic scale. (a), (e) Cross (B). (b), (f) Cross (T). (c), (g) Long (B). (d), (h) Long (T).

distribution for a given data set is the one that has the minimum $\mathrm{KL}$ and KS measures compared to the others. These results are shown in several sub-figures in Figures 4 and 5.

1) SAR Image Modeling: In Table III, the estimated distribution families and the resulting scale and shape parameters are shown for all seven SAR images. In addition, Table III presents the KL and KS significance test results for each SAR image. For urban images, both of the images follow generalized Rayleigh distribution with $p$-values of at least 0.9994 . These results provide further empirical evidence to the results of [3] and [2] which suggest generalized Rayleigh distribution to model urban scene SAR images.

Examining the forest scene SAR image results in Table III, we can state that forest images for both of the frequency bands have lighter tails than urban scenes, and the resulting distributions are $\mathcal{K}$ and Gamma. Please note that $\mathcal{K}$ and Gamma distribution families generally contain similar member distributions since $\mathcal{K}$ distribution is a compound/product distribution of Gamma. Both of the agricultural SAR images have similar histograms, whereas UHF-band forest scene SAR image has slightly fatter tails than that of X-band. For this type of histograms, due to having fatter tails than a $\mathcal{K}$ distribution, 
TABLE III

Modelling Results and Statistical Significance OF THE ESTIMATES FOR SAR IMAGES

\begin{tabular}{llccccc}
\hline Image & Est. & $\begin{array}{l}\text { Est. } \\
\text { Shape }(\hat{\alpha})\end{array}$ & $\begin{array}{l}\text { Est. } \\
\text { Scale }(\hat{\gamma})\end{array}$ & KL Div. & $\begin{array}{l}\text { KS } \\
\text { Score }\end{array}$ & $\begin{array}{l}\text { KS } \\
p \text {-value }\end{array}$ \\
\hline Urban-X & GenRayl & 1.6472 & 231.6267 & 0.0231 & 0.0280 & 1.0000 \\
Urban-L & GenRayl & 1.4488 & 94.9702 & 0.1020 & 0.0704 & 0.9994 \\
Forest-X & $\mathcal{K}$ & 7.8682 & 10.7058 & 0.0167 & 0.0163 & 1.0000 \\
Forest-UHF & Gamma & 2.1276 & 0.0313 & 0.0173 & 0.0247 & 1.0000 \\
Agricultural-X & Weibull & 3.3410 & 78.2370 & 0.0300 & 0.0363 & 1.0000 \\
Agricultural-L & Gamma & 5.5857 & 0.0780 & 0.0152 & 0.0317 & 1.0000 \\
Mixed-X & Gamma & 4.0309 & 0.0674 & 0.0234 & 0.0306 & 1.0000 \\
\hline
\end{tabular}

estimating Gamma distribution for UHF-band forest SAR image fulfills our prior expectations.

Analyzing the agricultural scene SAR image results in Table III, first, we can state that two images have characteristically different histograms and estimating different distributions for each has not been surprising. Thus, for the $\mathrm{X}$-band agricultural SAR image, the resulting distribution is a Weibull the shape parameter of which is higher than 3. This result shows that this image has nearly centralized intensity histogram. For a histogram of this type, estimating Weibull distribution provides evidence to a study in [15]. On the other hand, L-band agricultural scene SAR image seems to have a Rayleigh-type non-symmetrical distribution and the most suitable fitted distribution is a Gamma. For both forest and agricultural images, statistical significance results demonstrate remarkable fitting performance of the proposed method, all of which achieve a $p$-value of 1 .

Mixed scene image histogram is very similar to Forrest-UHF and Agricultural-L histograms with heavier tails. Since those images are modeled with the Gamma distribution, the estimated distribution family for the mixed scene image is also Gamma. Statistical significance values are very low and the estimation performance is also reasonable.

In Figure 4 estimated pdfs in both linear and logarithmic scales are shown for all seven SAR images. Logarithmic scales for each pdfs have been used to show the behavior of data for low probability regions. All sub-figures provide visual verification to the numerical results discussed above for Table III. Examining the figures, heavy-tailed characteristics of urban images are clear in the histograms. In addition, estimated generalized Rayleigh distributions generally follow the data histogram well. For both of the forest images, estimation performance is remarkable and estimated pdfs fit the data histogram for both low and high probability data ranges. Due to its nearly-centralized histogram, estimated distribution has slightly lower performance for the data histogram intensity values over 160 . However, for the lower intensities of Agricultural-X images and all intensities of Agricultural- $\mathrm{L}$ image, it is clearly seen that the estimated distributions fit the data histograms successfully.

Additionally, examining the Mixed- $X$ estimation results in Figure 4(m)-(n), it can be seen that the estimated distribution models the intensities around the peak of the histogram better rather than its tails. During the simulations, in a couple of 20 repetitions for mixed scene image, we have experienced that the proposed method estimates generalized
TABLE IV

Modelling Results and Statistical Significance of the ESTIMATES FOR US IMAGES. (B) AND (T) REFER TO BLOOD AND TISSUE SECTIONS, RESPECTIVELY

\begin{tabular}{lrlcclc}
\hline & $\begin{array}{r}\text { Est. } \\
\text { Family }\end{array}$ & $\begin{array}{l}\text { Est. } \\
\text { Shape }(\hat{\alpha})\end{array}$ & $\begin{array}{l}\text { Est. } \\
\text { Scale }(\hat{\gamma})\end{array}$ & KL Div. & $\begin{array}{l}\text { KS } \\
\text { Score }\end{array}$ & $\begin{array}{l}\text { KS } \\
\text { p-value }\end{array}$ \\
\hline Long (B) & $\mathcal{K}$ & 3.4342 & 0.1609 & 0.0112 & 0.0154 & 1.0000 \\
Long (T) & GenRayl & 1.1477 & 16.3052 & 0.0169 & 0.0470 & 1.0000 \\
Cross (B) & $\mathcal{K}$ & 1.8020 & 0.1818 & 0.0218 & 0.0335 & 1.0000 \\
Cross (T) & GenRayl & 0.9863 & 18.2985 & 0.0643 & 0.0926 & 0.9771 \\
\hline
\end{tabular}

Rayleigh distribution instead of Gamma. These trials model the tails of this image better rather than the intensities around the peaks resulting in higher $\mathrm{KL}$ and $\mathrm{KS}$ values than that of 0.0234 and 0.0306 , respectively. However, generally, in most of the repetitions, Gamma distribution has been estimated as the best matching distribution family.

2) Ultrasound Image Modeling: For US speckle modeling, we have studied four cases which are two US images and blood and tissue sections for each image. In Table IV, we show the estimation results for all four cases and their KL and KS test results, respectively. Examining the blood sections for both of the images, estimated pdfs are both $\mathcal{K}$ distribution. Both of the blood sections demonstrate Rayleigh-like (but subRayleigh) distribution characteristics and the proposed method estimates the most suitable distribution for blood sections. In addition, analyzing the tissue sections for both images, estimated pdfs are generalized Rayleigh for both of the images. Estimated distributions for all four cases have fulfilled the expectations and provide empirical evidence in support of the results of [5] and [9]-[11] that tissue sections have heavier tails than blood sections. In addition, generalized Rayleigh has shown better performance compared to Gamma and $\mathcal{K}$ distributions which are generally preferred distributions in the literature in modeling the heavy tailed characteristics of US images resulting from the speckle noise. For all the cases, statistical significance test results demonstrate the remarkable fitting performance of the proposed method by achieving $p$-values of at least 0.9771 . Results obtained from tissue section modeling provide further evidence to previous studies [10], [11] which propose generalized Rayleigh distribution for modeling of tissue sections.

The visual results for US speckle modeling in terms of linear and $\log$-scale pdfs are shown in Figure 5. Examining all the sub-figures, we can say that all the fitted distributions follow the data histograms well both for higher and lower probability ranges. Figure 5 provides visual support to the numerical results in Table IV. Particularly, Rayleigh-type characteristics of blood sections for both cross and longitudinal sections, has been clearly seen from the Figure 5-(a) and (c). The estimated distribution for these sections are $\mathcal{K}$ distribution and its performance is remarkable for both of the images and for all data range. Examining the tissue section results in Figure 5-(b), (d), (f) and (h), we can clearly see the heavy tailed characteristics of these sections. The lower probabilities are around $10^{-3}$ even for the maximum values. Estimated generalized Rayleigh distributions achieve great fitting performance for all data ranges of the tissue sections. 


\section{CONCLUSION}

In this study, we have proposed a generalized Bayesian model selection approach for remote sensing images based on trans-space RJMCMC in [21]. The proposed method in this paper, is an automatic statistical model class selection method among remote sensing images. Our method is clearly distinguished from other methods in the literature that focus on a single family at a time, since it is able to choose from various distribution classes. Moreover, the model class space can be easily adjusted according to the needs of the users, and thus the proposed method provides a very flexible use in various applications.

Particularly, the proposed method estimated generalized Rayleigh distribution for urban SAR and tissue section US images. These results are in good agreement with the studies in the literature and confirm the estimation performance of the proposed method. In addition, applicability of Gamma and $\mathcal{K}$ distributions for remote sensing images, land scenes of forest and agricultural SAR and blood pool sections of US images has been clearly demonstrated. In land scene SAR images with nearly-centralized histograms, Weibull distribution has been found as the suitable distribution model, and empirical evidence was provided to the studies in the literature.

During the simulations, we have experienced some similarities and differences between distribution families for different data sets. Particularly, for heavy tailed data sets such as Urban SAR and tissue US generalized Rayleigh is selected by RJMCMC and generally, intra-class-switch moves are accepted. This shows the success of the generalized Rayleigh distribution in modeling heavy tailed data sets. Additionally, for data sets which have lighter tails than mentioned images above (Forest SAR and Blood US), the proposed method has generally chosen Gamma or $\mathcal{K}$ distributions. Since $\mathcal{K}$ distribution is a compound and product distribution of the Gamma distribution, this result is expected and the algorithm frequently accepts inter-class-switch moves between $\mathcal{K}$ and Gamma distribution. During examining Rayleigh-like distributions, the proposed method is more likely to accept interclass-switch move between all the families, since Rayleigh is a common distribution for the most of the families. Because of this fact, selecting Rayleigh as the initial distribution, gives flexibility to the algorithm and speeds up the convergence. For the data sets which are less-skewed than a Rayleigh distribution (e.g. Agricultural-X), the proposed method often performs inter-class-switch moves between Weibull and Nakagami distributions rather than Gamma and $\mathcal{K}$ distributions.

Another success of the proposed approach for proposal distributions appears when there are lots of distributions whose statistical characteristics are very similar. The proposed usage can easily adjust itself for a transition between distributions the shape and scale parameters of which have extremely different values. In other words, matching the norms to calculate the parameters, offers to switch distributions the parameters of which are strictly different. This capability of the proposed method increases the number of model switches between similar distributions in presence of mimic phenomena. We have experienced this, especially between Gamma and $\mathrm{K}$ distributions. These two families are able to cover most of the members of each other. However, since the switched candidate distributions in each family have nearly the same statistical characteristics, this does not change the aim of this paper which is modeling the given data with the best (the most suitable) distribution. For further studies, this approach has possibility to open research directions to perform simulation studies about the mimicking capabilities of a distribution to another.

The presented approach can be used in a completely automated method for noise removal applications in remote sensing imaging methods. It can also be described as a method that can determine statistical models for remote sensing images other than the SAR and US. We would like to underline that the trans-space RJMCMC approach is not limited only to sampling across different distribution families but also can be extended to any class of models.

\section{REFERENCES}

[1] J. R. Eastman, "Guide to GIS and image processing," Clark Labs, Clark Univ., Worcester, MA, USA, Tech. Rep., 2001, vol. 1.

[2] A. Achim, E. E. Kuruoglu, and J. Zerubia, "SAR image filtering based on the heavy-tailed Rayleigh model," IEEE Trans. Image Process., vol. 15, no. 9, pp. 2686-2693, Sep. 2006.

[3] E. E. Kuruoglu and J. Zerubia, "Modeling SAR images with a generalization of the Rayleigh distribution," IEEE Trans. Image Process., vol. 13, no. 4, pp. 527-533, Apr. 2004.

[4] J. A. Noble and P. N. T. Wells, "Ultrasound image segmentation and tissue characterization," Proc. Inst. Mech. Eng. H, J. Eng. Med., vol. 224, no. 2, pp. 307-316, Feb. 2010.

[5] A. Osman and V. Kaftandjian, "Characterization of speckle noise in three dimensional ultrasound data of material components," AIMS Mater. Sci., vol. 4, no. 4, pp. 920-938, 2017.

[6] C. B. Burckhardt, "Speckle in ultrasound B-mode scans," IEEE Trans Sonics Ultrason., vol. SU-25, no. 1, pp. 1-6, Jan. 1978.

[7] P. Shankar et al., "Classification of breast masses in ultrasonic B scans using Nakagami and K distributions," Phys. Med. Biol., vol. 48, no. 14, p. 2229,2003

[8] V. Anastassopoulos, G. A. Lampropoulos, A. Drosopoulos, and M. Rey, "High resolution radar clutter statistics," IEEE Trans. Aerosp. Electron. Syst., vol. 35, no. 1, pp. 43-60, Jan. 1999.

[9] M. Alessandrini et al., "A pipeline for the generation of realistic 3D synthetic echocardiographic sequences: Methodology and open-access database," IEEE Trans. Med. Imag., vol. 34, no. 7, pp. 1436-1451, Jul. 2015.

[10] M. Pereyra and H. Batatia, "Modeling ultrasound echoes in skin tissues using symmetric $\alpha$-stable processes," IEEE Trans. Ultrason., Ferroelectr., Freq. Control, vol. 59, no. 1, pp. 60-72, Jan. 2012.

[11] M. Pereyra, N. Dobigeon, H. Batatia, and J.-Y. Tourneret, "Segmentation of skin lesions in 2-D and 3-D ultrasound images using a spatially coherent generalized Rayleigh mixture model," IEEE Trans. Med. Imag., vol. 31, no. 8, pp. 1509-1520, Aug. 2012.

[12] J. Jao, "Amplitude distribution of composite terrain radar clutter and the $\kappa$-distribution," IEEE Trans. Antennas Propag., vol. AP-32, no. 10, pp. 1049-1062, Oct. 1984.

[13] A. Lopes, E. Nezry, R. Touzi, and H. Laur, "Structure detection and statistical adaptive speckle filtering in SAR images," Int. J. Remote Sens., vol. 14, no. 9, pp. 1735-1758, Jun. 1993.

[14] L. Gagnon and A. Jouan, "Speckle filtering of SAR images: A comparative study between complex-wavelet-based and standard filters," Proc. SPIE, vol. 3169, pp. 80-91, Oct. 1997.

[15] M. Sekine and Y. Mao, Weibull Radar Clutter. Stevenage, U.K.: IET, 1990, no. 3.

[16] S. Kuttikkad and R. Chellappa, "Non-Gaussian CFAR techniques for target detection in high resolution SAR images," in Proc. IEEE Int. Conf. Image Process. (ICIP), vol. 1, Nov. 1994, pp. 910-914.

[17] B. Gambin and E. Kruglenko, "Temperature measurement by statistical parameters of ultrasound signal backscattered from tissue samples," Acta Physica Polonica A, vol. 128, no. 1, 2015. 
[18] H. Sportouche, J.-M. Nicolas, and F. Tupin, "Mimic capacity of fisher and generalized gamma distributions for high-resolution SAR image statistical modeling," IEEE J. Sel. Topics Appl. Earth Observ. Remote Sens., vol. 10, no. 12, pp. 5695-5711, Dec. 2017.

[19] V. A. Krylov, G. Moser, S. B. Serpico, and J. Zerubia, "Enhanced dictionary-based SAR amplitude distribution estimation and its validation with very high-resolution data," IEEE Geosci. Remote Sens. Lett., vol. 8, no. 1, pp. 148-152, Jan. 2011.

[20] P. J. Green, "Reversible jump Markov chain Monte Carlo computation and Bayesian model determination," Biometrika, vol. 82, no. 4, pp. 711-732, 1995

[21] O. Karakuş, E. Kuruoğlu, and M. Altınkaya, "Beyond trans-dimensional RJMCMC with a case study in impulsive data modeling," Signal Process., vol. 153, pp. 396-410, Dec. 2018.

[22] P. T. Troughton and S. J. Godsill, "A reversible jump sampler for autoregressive time series," in Proc. IEEE Int. Conf. Acoustics, Speech Signal Process., vol. 4, May 1998, pp. 2257-2260.

[23] R. S. Ehlers and S. P. Brooks, "Bayesian analysis of order uncertainty in ARIMA models," Federal Univ. Paraná, Curitiba, Brazil, Tech. Rep. 2004/05-B, 2004.

[24] S. Richardson and P. J. Green, "On Bayesian analysis of mixtures with an unknown number of components (with discussion)," J. Roy. Statist. Soc. B, Statist. Methodol., vol. 59, no. 4, pp. 731-792, 1997.

[25] D. Salas-Gonzalez, E. E. Kuruoglu, and D. P. Ruiz, "Modelling with mixture of symmetric stable distributions using Gibbs sampling," Signal Process., vol. 90, no. 3, pp. 774-783, 2010.

[26] O. Karakuş, E. E. Kuruoğlu, and M. A. Altınkaya, "Nonlinear model selection for PARMA processes using RJMCMC," in Proc. 25th Eur. Signal Process. Conf. (EUSIPCO), 2017, pp. 2110-2114.

[27] O. Karakuş, E. E. Kuruoğlu, and M. A. Altınkaya, "Bayesian volterra system identification using reversible jump MCMC algorithm," Signal Process., vol. 141, pp. 125-136, Dec. 2017.

[28] E. Conte and M. Longo, "Characterisation of radar clutter as a spherically invariant random process," IEE Proc. F-Commun., Radar Signal Process., vol. 134, no. 2, pp. 191-197, Apr. 1987.

[29] P. M. Shankar et al., "Classification of ultrasonic B-mode images of breast masses using Nakagami distribution," IEEE Trans. Ultrason., Ferroelectr., Freq. Control, vol. 48, no. 2, pp. 569-580, Mar. 2001.

[30] S. Ghofrani, M. Jahed-Motlagh, and A. Ayatollahi, "An adaptive speckle suppression filter based on Nakagami distribution," in Proc. EUROCON Trends Commun., Int. Conf., vol. 1, 2001, pp. 84-87.

[31] G. Moser, J. Zerubia, and S. B. Serpico, "SAR amplitude probability density function estimation based on a generalized Gaussian model," IEEE Trans. Image Process., vol. 15, no. 6, pp. 1429-1442, Jun. 2006.

[32] M. Nakagami, "The $m$-distribution-A general formula of intensity distribution of rapid fading," Statistical Methods in Radio Wave Propagations. New York, NY, USA: Pergamon, 1960, pp. 3-34.

[33] M. D. Yacoub, J. E. V. Bautista, and L. G. D. R. Guedes, "On higher order statistics of the Nakagami-m distribution," IEEE Trans. Veh. Technol., vol. 48, no. 3, pp. 790-794, May 1999.

[34] D. R. Iskander and A. M. Zoubir, "Estimation of the parameters of the K-distribution using higher order and fractional moments [radar clutter]," IEEE Trans. Aerosp. Electron. Syst., vol. 35, no. 4, pp. 1453-1457, Oct. 1999.

[35] Z. Tao, H. D. Tagare, and J. D. Beaty, "Evaluation of four probability distribution models for speckle in clinical cardiac ultrasound images," IEEE Trans. Med. Imag., vol. 25, no. 11, pp. 1483-1491, Nov. 2006.

[36] C. Walck, "Hand-book on statistical distributions for experimentalists," Part. Phys. Group, Stockholm Univ., Stockholm, Sweden, Tech. Rep. SUF-PFY/96-01, 1996.

[37] G. Gao, K. Ouyang, Y. Luo, S. Liang, and S. Zhou, "Scheme of parameter estimation for generalized gamma distribution and its application to ship detection in SAR images," IEEE Trans. Geosci. Remote Sens., vol. 55, no. 3, pp. 1812-1832, Mar. 2017.

[38] O. Garcia, "Simplified method-of-moments estimation for the Weibull distribution," New Zealand J. Forestry Sci., vol. 11, no. 3, pp. 304-306, 1981.

[39] G. Tzeremes and C. Christodoulou, "Use of Weibull distribution for describing outdoor multipath fading," in Proc. IEEE Antennas Propag. Soc. Int. Symp., vol. 1, Jun. 2002, pp. 232-235.

[40] T.-Y. Hwang and P.-H. Huang, "On new moment estimation of parameters of the gamma distribution using its characterization," Ann. Inst. Statist. Math., vol. 54, no. 4, pp. 840-847, 2002.

[41] W. W. Piegorsch and G. Casella, "The existence of the first negative moment," Amer. Statistician, vol. 39, no. 1, pp. 60-62, 1985.
[42] M. I. Skolnik, "Introduction to radar," in Radar Handbook, vol. 2. New York, NY, USA: McGraw-Hill, 1962.

[43] Artemis Inc. Synthetic Aperture Radar Solutions. Accessed: Sep. 2017. [Online]. Available: http://artemisinc.net/media.php

[44] H. Liebgott, A. Rodriguez-Molares, F. Cervenansky, J. A. Jensen, and O. Bernard, "Plane-wave imaging challenge in medical ultrasound," in Proc. IEEE Int. Ultrason. Symp. (IUS), Sep. 2016, pp. 1-4.

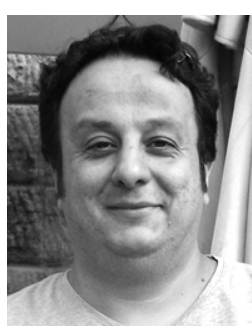

Oktay Karakuş received the B.Sc. degree (Hons.) in electronics engineering from Istanbul Kültür University, Turkey, in 2009, and the M.Sc. and Ph.D. degrees in electronics and communication engineering from the Izmir Institute of Technology, Turkey, in 2012 and 2017, respectively. From 2009 to 2017, he was with the Department of Electrical and Electronics Engineering Yasar University, Turkey, and the Department of Electronics and Communication Engineering, Izmir Institute of Technology, as a Research Assistant. Since 2018, he has been a Research Associate in image processing with the Visual Information Laboratory, Department of Electrical and Electronic Engineering, University of Bristol. His research interests are mostly in statistical/Bayesian signal and image processing and its applications in nonlinear system modeling, remote sensing, telecommunications, and energy sources.

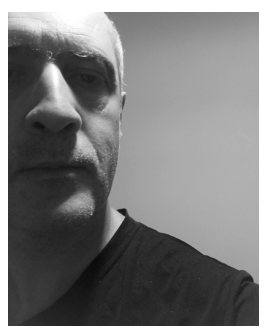

Ercan E. Kuruoğlu (M'98-SM'06) received the M.Phil. and Ph.D. degrees in information engineering from the University of Cambridge, Cambridge, U.K., in 1995 and 1998, respectively. In 1998, he joined the Xerox Research Center Europe, Cambridge. He was an ERCIM Fellow with INRIA-Sophia Antipolis, France, in 2000. In 2002, he joined ISTI-CNR, Pisa, Italy, where he is currently a Senior Researcher in telecommunications and computer science with a focus on habilitation degree. He was a Visiting Professor with Georgia Tech, China, in 2007, 2011, and 2016. He was a Chinese Government 111 Project Foreign Expert from 2007 to 2011. His research interests are in the areas of statistical signal and image processing and machine learning with applications in computational biology, telecommunications, and remote sensing. $\mathrm{He}$ is a member of the IEEE Technical Committee on Signal Processing Theory and Methods and the EURASIP Special Area Team in biomedical signal and image analytics and machine learning. He was an Alexander von Humboldt Experienced Research Fellow of the Max Planck Institute for Molecular Genetics from 2013 to 2015. He was the Technical Co-Chair of EUSIPCO 2006 and the Tutorial Co-Chair of ICASSP 2014. $\mathrm{He}$ was an Associate Editor of the IEEE TRANSACTIONS ON Signal Processing and the IEEE Transactions on ImAge Processing. He is currently the Editor-in-Chief of Digital Signal Processing: A Review Journal. He was a Tutorial Speaker at the IEEE ICSPCC 2012. He was a plenary speaker at ISSPA 2010, the IEEE SIU 2017, and Entropy 2018.

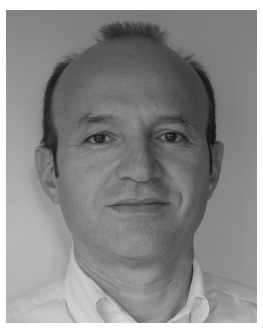

Mustafa A. Altinkaya (M'02) received the B.S., M.S., and Ph.D. degrees in electrical and electronics engineering from Bogazici University, Istanbul, Turkey, in 1987, 1990, and 1996, respectively. From 1989 to 1990, he was a Research Associate with the TUBITAK Marmara Research Center, Kocaeli, Turkey. From 1991 to 1996, he was a Research and Teaching Assistant with Bogazici University. He was a part-time Instructor in Air Force Academy, Naval Academy, and Bogazici University, from 1995 to 1998. In 1998, he joined the Electrical and Electronics Engineering Department, İzmir Institute of Technology, where he is currently an Associate Professor. His research interests are mainly on statistical signal processing and its applications in telecommunications, wireless networks, and positioning and structural dynamic systems. He is currently an Associate Editor of the Turkish Journal of Electrical Engineering and Computer Sciences. 